\title{
Ein fruktifizierender Sproß von Asolanus camptotaenia Wood aus dem Westfal D Nordwestdeutschlands
}

\author{
Stephan Schultka ${ }^{1}$ \\ Mit 2 Tafeln und 2 Abbildungen
}

\section{Kurzfassung}

Erstmalig wird der fruktifizierende Sproß von Asolanus camptotaenia beschrieben. Bei den nachgewiesenen fertilen Organen handelt es sich um Megasporangien, Microsporangien sind noch nicht gefunden worden. Der Bau des fertilen Sprosses sowie die Organisation der Sporophylle belegen die isolierte Stellung der Gattung Asolanus innerhalb der Lepidophyta.

Schlüsselwörter: Asolanus, Lepidophyta, Oberkarbon, Piesberg, Fruktifikation, Nordwestdeutschland

\begin{abstract}
A megasporangia bearing axis of Asolanus camptotaenia Wood from the Westphalian D of Northwestern Germany

A fertil impression axis of Asolanus camptotaenia from Westfalian D strata is described. While some megasporangia are found, microsporangia are not yet established. The type specimen is not very well characterized by Wood 1860 , but all differences described til now would be caused by preservation. The diagonal striation from cushion to cushion also is only a special mode of preservation and useless for taxonomic purposes. Well sculptered Asolanus-axis from the Stephanian belong to the genus Lepidodendron or possibly to a new not yet described genus. Lepidostrobus foliaceus and Lepidophyllum truncatus mentioned by Lesquereux 1880 as well as Gymnostrobus salisbury described by Bureau 1914 may belong to A. camptotaenia.

The organisation of the fertil axis from the Piesberg demonstrates an isolated position of this genus within the lepidophytes not so far from Pinakodendron and relatives.
\end{abstract}

Key words: Asolanus, Lepidophyta, upper Carboniferous, Piesberg, fructification, northwestern Germany

\section{Einleitung}

Asolanus ist eine monospezifische Gattung innerhalb der Lepidophyten, die allgemein recht selten ist. Nur im höheren Karbon wird sie häufiger erwähnt bzw. als häufig angegeben (u.a. Zeiller 1888 , Josten 1991). Bisher wurden nur unverzweigte Achsen mit locker gestellten, querrhombiodalen Blattpolstern beschrieben, die in der oberen Hälfte ebenfalls querrhomboidale Blattnarben mit einer einfachen, ungegliederten, rundlich-ovalen Marke tragen. Die Blattpolster sind durch eine typische, diagonal verlaufende, feine Streifung verbunden. Zusammen mit weiteren Formgattungen unklarer systematischer $\mathrm{Zu}$ ordnung wie Bothrodendron und Pinakodendron steht Asolanus relativ isoliert in der Gruppe der Lepidophyten. Eine Verwandtschaft von Asola- nus mit der Gattung Lepidodendron wird von den meisten Autoren aufgrund des Baus der Blattnarbe sowie dem Auftreten von KnorriaErhaltungszuständen angenommen. Außer $A$. camptotaenia sind nach Kidston (1911: 175) und Chaloner (in Boureau 1967: 641) alle anderen bisher beschriebenen Arten als nicht ausreichend begründet anzusehen. Fertile Organe im Zusammenhang mit Asolanus wurden bisher nicht beschrieben.

In älteren Aufsammlungen vom Piesberg sind Lepidophytenreste nicht selten anzutreffen, vor allem die Gattung Asolanus wird von dieser Fundstelle ausführlich beschrieben (Weiss \& Sterzel 1893). Die intensiven Aufsammlungen in den letzten 20 Jahren über allen aufgeschlossenen Flözen am Piesberg (Abb. 1) ergaben jedoch nur wenige Lepidophytenreste, die annähernd

${ }^{1}$ Museum für Naturkunde, Institut für Paläontologie, Invalidenstraße 43, D-10115 Berlin, Germany. e-mail: stephan schultka@rz.hu-berlin.de Erhalten Februar 1998, angenommen Juni 1998 


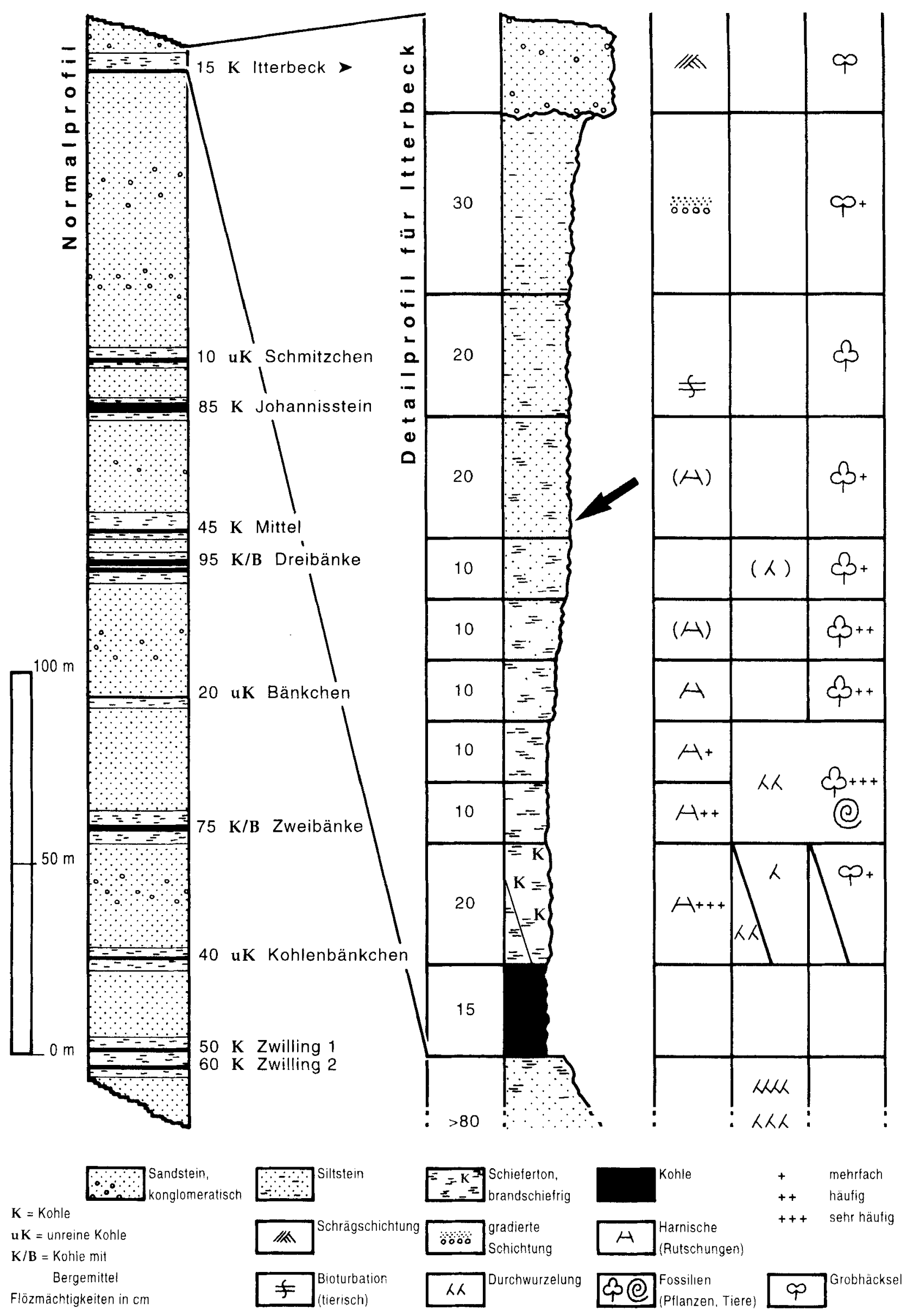


bestimmt werden konnten (Josten et al. 1984 und eigene Beobachtungen), was im deutlichen Gegensatz zu den Funden in den älteren Schichten des Ruhrgebietes und von Ibbenbüren steht. Nur im Hangenden von Flöz Itterbeck, dem jüngsten Flöz in der Piesberg-Abfolge, erbrachten Detailaufnahmen gehäuft Funde von Lycopsiden. Dabei handelt es sich fast ausschließlich um beblätterte Äste von Lepidodendron ophiurus Brongniart. Wenige andere Achsenbruchstücke von Lycophyten sind der Gattung Asolanus Wood zuzuordnen. Erstmalig konnten an einem dieser Achsenreste mehrere fertile Organe nachgewiesen werden.

\section{Erhaltung}

Unter den Funden von Asolanus camptotaenia aus dem Hangenden von Flöz Itterbeck befindet sich ein einzelnes, fertiles, etwa $12 \mathrm{~cm}$ langes und $7 \mathrm{~cm}$ breites Achsenbruchstück. Es liegt in Abdruckerhaltung (impressions) vor. Alle organischen Reste sind in Gümbelit umgewandelt, der durch Verwitterungsprozesse zum großen Teil fortgeführt oder in Eisenhydroxide umgewandelt wurde.

Auf derselben Oberfläche finden sich häufiger nur schemenhaft ausgebildete Blattpolster direkt neben kräftig skulpturierten. Eingesenkte Polster stehen neben deutlich erhaben hervorstehenden, sie sind oftmals gegeneinander verschoben und können sich sogar überschneiden (Taf. 1: 2). Diese Erhaltungsweise erwähnt auch Chaloner (in Boureau 1967: 642). Sie kann darauf zurückgeführt werden, daß die Blattpolster z.T. von der Gegenseite durchgedrückt wurden.

In der Mitte der Achse ist eine $10 \mathrm{~mm}$ breite, in Längsrichtung verlaufende Streifung zu erkennen. Ähnliche Streifungen sind auch bei den sterilen Achsenbruchstücken von $A$. camptotaenia nachweisbar (Taf. 1: 2). Eine vergleichbare Streifung bilden Gothan \& Remy (1957: 94) bei einem Stammstück von $A$. camptotaenia von der Zeche Baldur/Dorsten ab (lt. beiliegendem Etikett Flöz 18, Westfal C). Wie schon Koehne in Potonié (1904: 5) feststellte, handelt es sich dabei um den nach außen durchgedrückten Holzkörper.
Um bei dem fertilen Stammstück die ansitzenden Sporophylle im Sediment freizulegen, müssen erst diese Struktur bzw. die Reste der Rinde mit den Blattpolstern abgetragen werden. Der Betrachter blickt somit auf die Innenseite der Rindengewebe bzw. die adaxiale Seite der Sporophylle, die von der Achse weg etwas ins Sediment ragen.

Die Sporophylle sind leicht gewölbt, wodurch sie bei der Einbettung in das Sediment häufig einrissen und wellig verfaltet wurden. Die ebenfalls vorkommenden, typischen LepidophytenBlätter sind oft verknickt und meist nur noch als Schleier auf der Schichtfläche erhalten und daher schwer präparativ freizulegen. Sie waren offensichtlich bereits vor der Einbettung zum Teil abgebaut. Bei dem vorliegenden Material handelt es sich somit um Abdrücke nicht entrindeter Stücke, die längere Zeit im Wasser flotierten, bevor sie eingebettet wurden.

\section{Systematischer Teil}

\section{Asolanus camptotaenia Wood}

Material: 3 Achsen, davon eine fertil, Inv.-Nr. 1996/313a u. b, 1996/314a u. b

Fundort: Mülldeponie Piesberg bei Osnabrück (ehem. Südbruch der Klöckner-Durilit $\mathrm{GmbH}$ )

Alter: Hangendes von Flöz Itterbeck, Westfal D

Beschreibung

Das etwa $12 \mathrm{~cm}$ lange und $7 \mathrm{~cm}$ breite fertile Achsenbruchstück ist dicht mit Sporophyllen besetzt. Diese stehen in einer flachen Spirale an der Achse und sitzen so dicht über- und nebeneinander, daß sie sich gegenseitig überlappen (Taf. 1: 1).

Die Sporophylle sind etwa $16 \mathrm{~mm}$ lang, bei einer Breite von etwa 6,5 mm (Taf. 1: 3). Die Seitenränder sind leicht wulstig verdickt, etwas gewellt und im mittleren Abschnitt weitgehend parallel. Die Spitze ist etwas ausgezogen und stumpf gerundet. Die Basis der Sporophylle erscheint in vielen, aber nicht in allen Fällen von der eigentlichen Blattspreite etwas abgesetzt zu sein und knickt zum Stamm hin ab, wie dies von den Sporophyllen der Lepidostroben bekannt ist. So entsteht ein Schild-förmiger Bereich, der tra-

Abb. 1. Normalprofil vom Piesberg (modifiziert nach Knauff, W., Köwing, K. \& Rabitz, A. 1971) und Detailprofil über Flöz Itterbeck, Pfeil weist auf Fundlage mit Asolanus camptotaenia hin

Fig. 1. Generalized profile of the Piesberg-quarry (modified from Knauff, W., Köwing, K. \& Rabitz, A. 1971) and the detailed profile of the Itterbeck seam, arrow points to the layer with Asolanus camptotaenia 

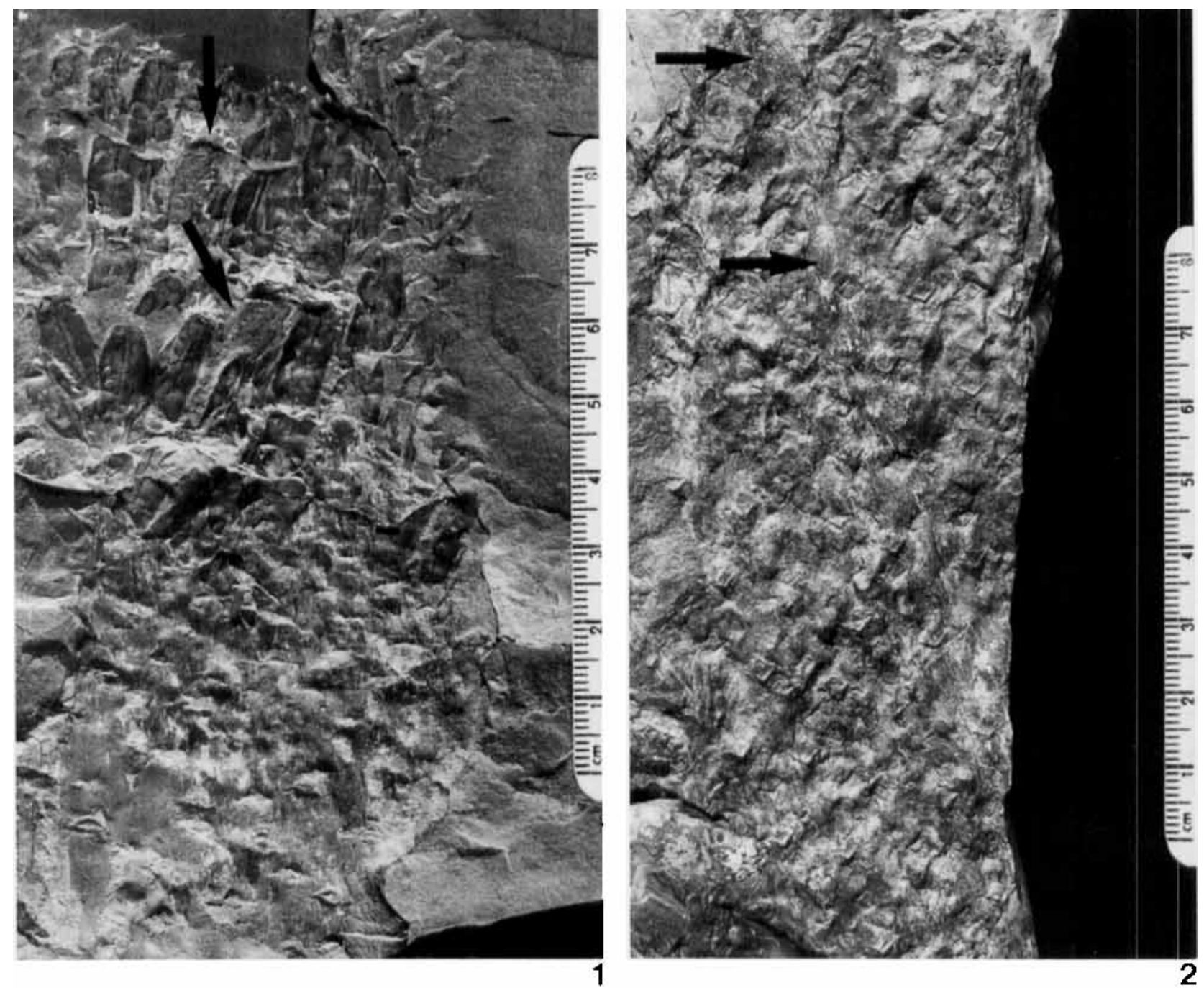

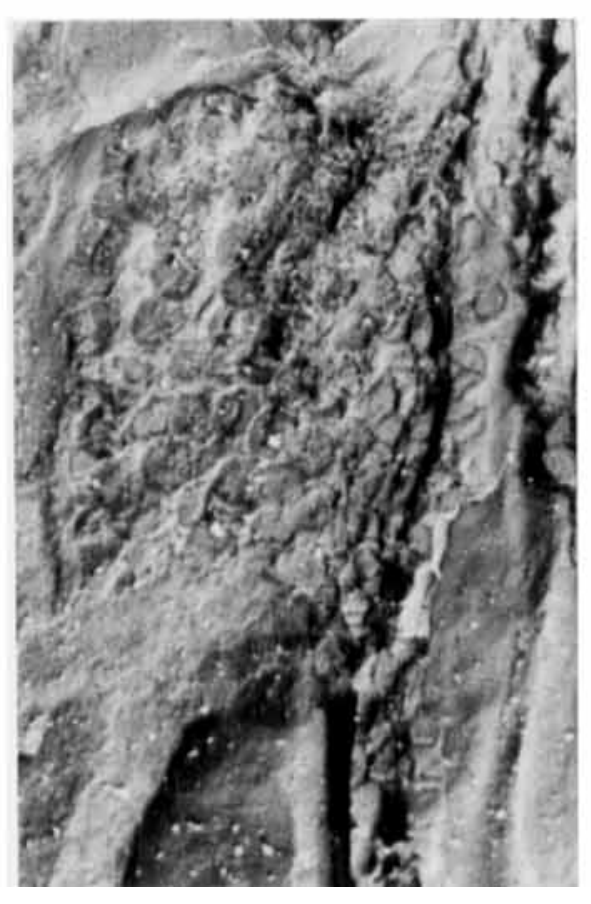

4

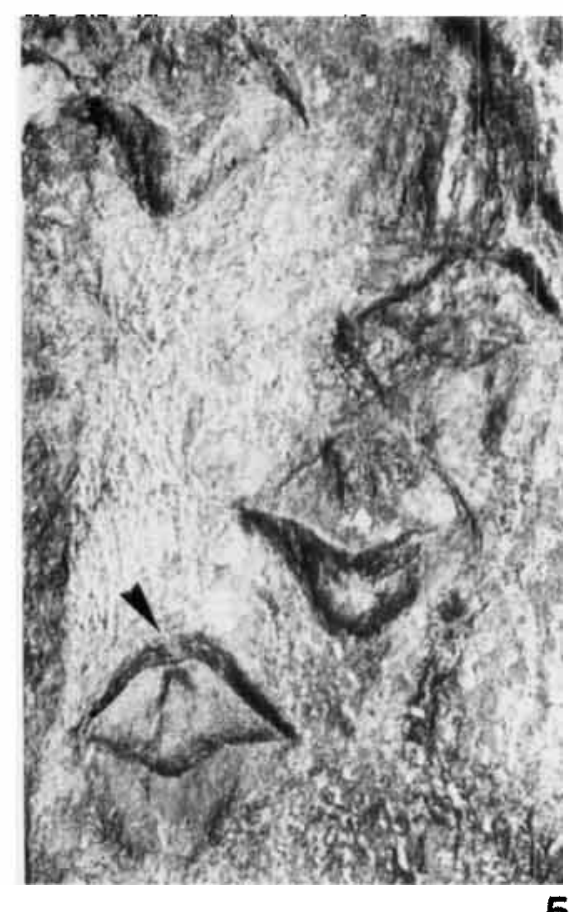




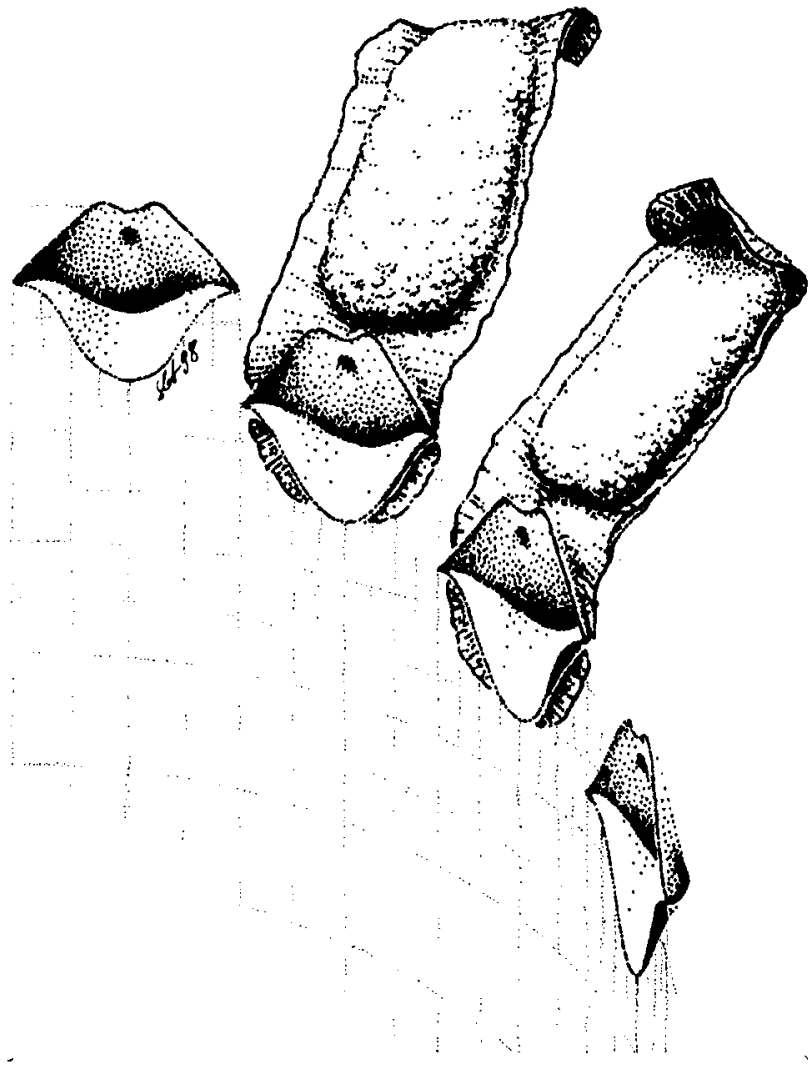

Abb. 2. Abstrahierte Rekonstruktion der Sporophylle von $A$. camptotaenia mit den ansitzenden Megasporangien

Fig. 2. Reconstruction of the A. camptotaenia sporophylls with attached megasporangia

pezförmig gerundet, durchschnittlich $3,4 \mathrm{~mm}$ hoch ist und den Blattgrund bzw. das Pedicel bildet. Er verbreitert sich kontinuierlich von der tragenden Achse weg bis zur maximalen Breite der Lamina des Sporophylls. Im Bereich des Umknickens reißt die Spreite der Lamina leicht ein, so daß häufig an dieser Stelle ein breit gerundetes, herzförmiges Einziehen vorgetäuscht wird. Bei manchen Sporophyllen kann eine so ausdifferenzierte Basis nicht nachgewiesen werden, was auf unterschiedliche Erhaltungsbedingungen zurückgeführt werden muß. Etwa $3 \mathrm{~mm}$ vor der stumpf gerundeten Spitze des Sporophylls knickt die Spreite oftmals abaxial um, einige Male ist sie auch adaxial umgebogen (Abb. 2). Auch hier ist im Bereich des Um- knickens der Spreitenrand häufig eingerissen und kann eine morphologische Abtrennung von der Lamina vortäuschen.

Gelegentlich ist das Pedicel in einen basalen, etwas zungenförmigen Teil und einen distalen etwas querrhombisch geformten Teil gegliedert, weitere Differenzierungen sind nicht erkennbar. An der Grenze zwischen Pedicel und nach außen umknickender Sporophyllspreite ist eine deutliche, längsovale bis kreisrunde Marke entwickelt, die einen Durchmesser zwischen 0,6 und $1 \mathrm{~mm}$ aufweist (Taf. 2: 3). Diese Marke ist in ihrem inneren Bereich nicht glatt. Es liegt daher der Verdacht nahe, daß es sich um eine Abrißmarke handelt. Möglicherweise markiert sich hier das Leitbündel beim Eintritt in das Sporangium („Leitbündelmarke“).

Die auffallend breite und sehr kompakte Mittelader der Lamina mißt an der Eintrittsstelle bis zu 0,9 mm in der Breite. Zum distalen Ende hin wird sie sehr langsam schmaler und endet kurz vor Erreichen des Vorderrandes (Taf. 1: 3). Teilweise verschwindet sie kontinuierlich, manchmal scheint sie aber auch relativ abrupt zu enden. Sie ist auf der adaxialen Seite der Lamina etwas eingesenkt und tritt auf der Gegenseite entsprechend etwas erhaben hervor. Die gesamte Oberfläche der Lamina weist eine feine Streifung auf, die weitgehend senkrecht auf dem äußeren Rand des Sporophylls steht (Taf. 2: 4). Sie wird von der Anordnung der Epidermiszellen hervorgerufen.

An insgesamt vier Stellen wurden auf den an der Achse ansitzenden Sporophyllen Megasporen nachgewiesen. Freigelegt zeigen sich dicht zusammenhängende Megasporenmassen von abgerundet-rechteckigem Umriß, die etwa $10 \mathrm{~mm}$ Länge und $6 \mathrm{~mm}$ Breite erreichen (Taf. 1: 4; Taf. 2: 1,2). Damit bedecken sie fast vollständig den gesamten mittleren Bereich der Lamina zwischen dem Pedicel und dem abknickenden Spitzenbereich. Sie sind jeweils entlang der Längsachse der Sporophyllen ausgerichtet und liegen auf der adaxialen Seite der Lamina. Reste der Sporangienwand sind nicht eindeutig zu erken-

Taf. 1. 1. An einer Achse ansitzende Sporophyllen (Inv.-Nr. 1996/313), basal Achse mit querrhombischen Blattpolstern erkennbar (Pfeile weisen auf Megasporangien hin). 2. Rückseite der Achse (Inv.-Nr. 1996/313) mit zentralem Holzkörper (Pfeil) und Blattpolstern mit positivem wie negativem Relief. 3. Sporophyll mit kräftiger Mittelader und deutlicher Streifung der Lamina, 5:1. 4. Megasporenhaufen auf einem Sporophyll (Position siehe Pfeil auf Fig. 1), bedampft, $7: 1$. 5. Querrhombisches Blattpolster mit Leitbündelstutzen, Pfeil weist auf vermutete Position der Ligulargrube (Inv.-Nr. 1996/314), $5: 1$

Plate 1. 1. Sporophylls attached at an axis (Inv.-Nr. 1996/313) with the typical transvers rhombic cushions (arrows point to megasporangia). 2. Back of axis (Inv.-Nr. 1996/313) with central vascular cylinder (arrow) and positively or negatively embossed cushions. 3. Sporophylls mit clearly marked central vein and well developed striation on the lamina, $5: 1$. 4. Megaspores on a sporophyll (position see arrow on fig. 1), $7: 1$. 5. Transvers rhombic leaf cushion with vascular bundle, arrow points to supposed position of ligule pit (Inv.-Nr. 1996/314), $5: 1$ 

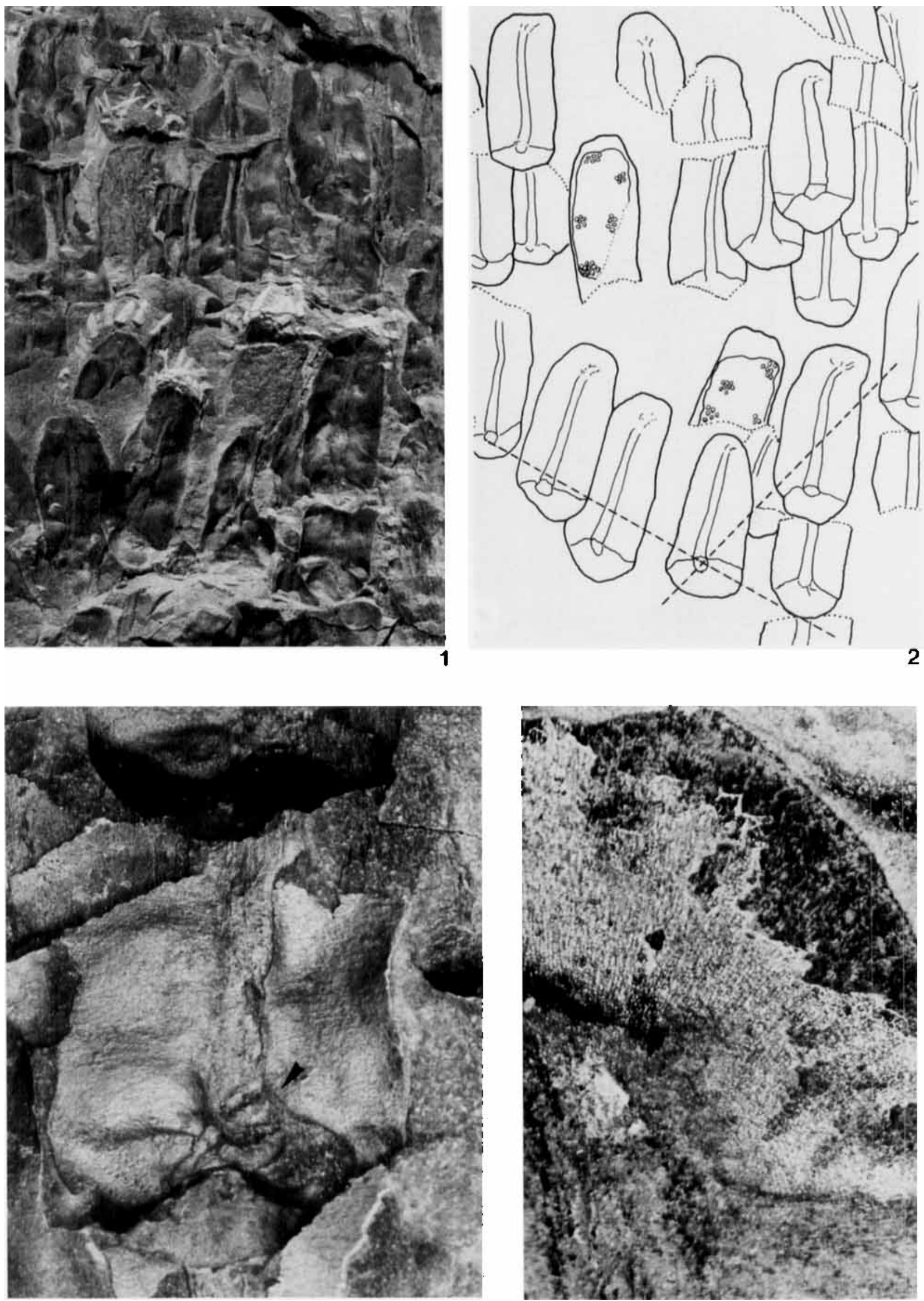
nen, können aber vermutet werden. Die konstante Form sowie die Ausrichtung zur Längsachse in Verbindung mit der immer genau mittigen Position auf den Sporophyllen schließt eine zufällige Einschwemmung sowie der Eintrag durch Tiere, z. B. sporenfressende Arthropoden, aus.

Die einzelnen Megasporen sitzen zumindest teilweise noch in Tetraden zusammen, d. h. es liegen offensichtlich unreife Sporangien vor. Der Durchmesser der einzelnen Megasporen liegt zwischen 0,4 und $0,6 \mathrm{~mm}$ (Taf. 1: 4). Die Tetradenmarke erreicht fast den Rand, der möglicherweise verstärkt ist. Eine schwache Punktierung der Oberfläche deutet auf eine Ornamentierung hin, weitere Einzelheiten sind beim vorliegenden Erhaltungszustand nicht erkennbar.

Etwa $10 \mathrm{~cm}$ weiter im Liegenden des Profils konnten einige isolierte Megasporenmassen aufgesammelt werden. Die drei eng nebeneinanderliegenden Sporenanhäufungen weisen ebenfalls einen abgerundet-rechteckigen Umriß auf. So stimmen sie in der äußeren, morphologischen Erscheinung weitgehend mit den Megasporenansammlungen auf dem oben beschriebenen Sproßteil überein. Das heißt, daß die Megasporangien mit sehr widerstandsfähigen Wänden ausgestattet waren, die einen Transport vollständiger Sporangien über einen längeren Zeitraum hinweg möglich machten.

Auf der Oberfläche der fertilen Achse ist eine Vielzahl von Blattnarben sichtbar, die die Merkmale von $A$. camptotaenia erkennen lassen. Sie stehen oft sehr unregelmäßig, was auf Verdrückungen und das Durchpausen der Polster von der Unterseite des Sproßstückes im Verlauf der taphonomischen Prozesse zurückzuführen ist. Die Blattnarben sind zwischen 4,0 und $4,5 \mathrm{~mm}$ breit bei einer Höhe von 2,3 bis $2,8 \mathrm{~mm}$ (Taf. 1:1). Sie haben somit den für das Genus Asolanus typischen, querrhombischen bis linsenförmigen Umriß mit sehr spitz auslaufenden Seiten-ecken, im Gegensatz zu den sehr breit gerundeten, oberen und unteren Ecken (Taf. 1: 5). Es treten zwei ungleiche Hauptparastichen mit Steigungswinkeln von etwa $130^{\circ}$ und $30^{\circ}$
(Längsachse der Stammstücke $=90^{\circ}$ ) auf (Taf. 2: 1, 2). Der Abstand der Parastichen untereinander schwankt um $10 \mathrm{~mm}$. Zum distalen Ende hin verlaufen die Parastichen auf dem fertilen Stammstück steiler, bei gleichzeitiger Verringerung des Abstandes der Parastichen untereinander (Stauchung). Der Abstand zwischen den einzelnen Polstern innerhalb einer Parastiche ist variabel, meist liegt er bei $10 \mathrm{~mm}$, gemessen von Leitbündelmarke zu Leitbündelmarke. Auf dem fertilen Stammstück scheinen die Abstände geringer zu sein, dort kann man oft nur einen $\mathrm{Ab}$ stand von 6 bis $7 \mathrm{~mm}$ messen.

Der obere Rand der einzelnen Blattnarben zeigt häufig median eine Einbuchtung, die einen V-förmigen Umriß aufweist (Taf. 1: 5). Im Blattnarbenbereich selbst ist die Erhaltung fast durchgehend sehr schlecht, worauf auch immer wieder in der Literatur hingewiesen wird. Gut erkennbar ist eine ovale, oft auch umgekehrt eiförmige, meist im Bereich des oberen Blattnarbenbereiches liegende erhabene Marke. Eine Differenzierung innerhalb dieser Marke ist nicht erhalten.

Um die eigentliche Blattnarbe herum befindet sich ein weitgehend glattes bis schwach gestreiftes, hochgewölbtes Feld, das dem Blattpolster bei den Lepidodendren entsprechen dürfte. Dieses Blattpolster umschließt die Blattnarbe weitgehend, die ausgezogenen, spitzen Seitenecken der Blattnarbe erreichen mehrfach knapp den äußeren Rand der Blattpolster. Die Gesamthöhe des Blattpolsters beträgt etwa 4,5 bis $5,5 \mathrm{~mm}$, die Breite schwankt zwischen 4,5 und 5,3 mm.

Der Blattpolster-Bereich über der Blattnarbe überschreitet nur selten die Höhe von $0,5 \mathrm{~mm}$, verläuft weitgehend parallel und fällt sehr steil zur Basis des Blattpolsters auf der Achsenoberfläche ab (Taf. 1: 5). Der Bereich unterhalb der Blattnarbe wird dagegen über $2 \mathrm{~mm}$ hoch. Er erscheint insgesamt rundlich-dreieckig bis zungenförmig und endet nach unten hin breit gerundet. Keine der Blattpolster weisen so deutlich schlanke, wulstförmig ausgezogene, längsgestreckte, hochgewölbte und nach unten hin langsam ausklingende Bereiche auf, wie sie z. B. Weiss \& Sterzel (1893: Taf. 4: 23, 24; Taf. 5: 28) aus dem Saarrevier abbilden.

Taf. 2. 1. Ausschnitt aus Taf. 1: 1 (Inv.-Nr. 1996/313), 2:1. 2. Zeichnerische Darstellung von Taf. $2: 1$ im gleichen Maßstab, gestrichelte Linien deuten die Parastichen an. 3. Basaler Bereich eines Sporophylls mit kräftigem, zentralem Leitbündel und rundlicher Leitbündelmarke (Pfeil), Ausschnitt aus Taf. 1: 1, 10:1. 4. Vorderrand eines Sporophylls mit senkrecht auf den Rand auftreffenden Zelireihen, Ausschnitt aus Taf. 1: 1, unter Immersion, 30:1

Plate 2. 1. Part of pl. 1: 1, (Inv.-Nr. 1996/313), 2: 11. 2. Drawing of pl. 2: 1, same scale, dashed lines outline the parastichs. 3. Basal part of sporophyll with big central vein and rounded outlet of vascular bundel (arrow), part of pl. 1: 1, 10:1. 4. Rows of cells meeting rectangular front of sporophyll, part of pl. 1: 1, under immersion, $30: 1$ 
Im Museum für Naturkunde, Berlin, liegt ein sehr gut erhaltenes Asolanus-Stammstück vom Piesberg (Gegendruck zu Weiss \& Sterzel 1893: Taf. 4: 25), das den Bau der Blattnarben noch deutlicher zeigt. Zentral auf der Blattnarbe ist eine rundliche Vertiefung angelegt, in deren Mitte sich abgerundet dreieckig der Leitbündelstutzen erhebt. Am oberen Rand der Marke deutet ein länglicher, sich nach oben verbreiternder Wulst die Lage einer Ligula an. Die Oberfläche der Blattpolster zeigt ein großlumiges, sehr kräftiges Zellmuster. Irgendwelche Hinweise auf das Vorliegen von Parichnosmalen im Bereich der Blattnarbe wie des gesamten Blattpolsters fehlen vollständig. Einen vergleichbaren Bau der Blattnarben beschreibt bereits Zeiller (1888: 588).

\section{Diskussion}

Die vorliegenden Stücke entsprechen in der Ausbildung der Blattnarben grundsätzlich dem von Wood (1861, nach Crookall 1964) abgebildeten, aber nicht sehr ausführlich beschriebenen Typus von $A$. camptotaenia. Unterschiede im Bau der Blattpolster, die sich bei den von Weiss \& Sterzel (1893) abgebildeten und beschriebenen Stücken zeigen, dürften auf unterschiedliche Erhaltungszustände innerhalb des Rindengewebes zurückzuführen sein.

So erscheint bei den Abbildungen von Wood (1861, 1869) der untere Teil des Blattpolsters mehr abgerundet-dreieckig bzw. gedrungen zungenförmig, wodurch der gesamte Blattansatz sehr breit gedrungen und kompakt erscheint. Vergleichbare Blattpolster bilden Roehl (1868), Zeiller (1906), Gothan \& Remy (1957), Vogellehner (1967) und andere ab. Viele Stücke weisen aber auch Blattpolster auf, die unter der Blattnarbe als langgezogener, hochgewölbter, schmaler Wulst ausgebildet sind, wodurch die Blattansätze insgesamt einen schmaleren, langgestreckten Eindruck machen. So können auch Stücke vom Piesberg diesen Blattpolstertyp zeigen (Weiss \& Sterzel 1893: Taf. 4: 22, 25). Sehr deutlich scheint diese Erhaltung bei dem nur unzureichend von Roemer (1864: Taf. 8: 12) abgebildeten Lepidodendron barbatum (=Asolanus camptotaenia nach Hörich 1920) vom Flöz Mittel (Piesberg) zu sein.

Dieser Erhaltungszustand stellt den Übergang zur Knorria-Bildung dar, wie sie beim Entrinden von Asolanus-Stammstücken entsteht. Dies wird u. a. auch bei dem Gegenstück zum Original von
Weiss \& Sterzel (1893) deutlich, wo beide Erhaltungszustände auf einem Stück überliefert sind. $\mathrm{Da} \beta$ es sich tatsächlich um Erhaltungszustände der gleichen Pflanze handelt, zeigt auch die Beobachtung, daß die Blattnarben immer undeutlicher werden, je stärker die langausgezogenen, hochgewölbten Blattpolster ausgebildet sind.

Viele Autoren führen als typisch und artbestimmend die diagonal von Blattpolster zu Blattpolster verlaufende, meist feine aber deutliche Streifung an, die auf der Stammoberfläche von Asolanus ein rautenförmiges Muster erzeugt. Sie ist auf einen bestimmten, häufigen Erhaltungszustand zurückzuführen, worauf bereits Crookall (1964: 349) hinweist. Diese Skulpturierung ist daher als Merkmal zur Definition der Gattung ungeeignet und sollte bei der Beschreibung der diagnostischen Merkmale von Asolanus nicht mehr erscheinen, zumal dieser Erhaltungszustand z.B. bei der Gattung Pinakodendron ebenfalls auftreten kann (Amerom \& Gaipl 1991: 221, Abb. 8). Es muß daher eher als ein diagnostisches Merkmal der gesamten Lepidophytengruppe um Asolanus angesprochen werden.

Gelegentlich treten zu Asolanus gestellte Stücke auf, die eine sehr kräftige, fast schon wulstige Skulpturierung der Stammoberfläche zwischen den Blattpolstern aufweisen. Solche Formen wurden bisher nur aus dem Stefan beschrieben (Vetter 1968, Daber \& Kahlert 1970). Die systematische Stellung dieser Stücke erscheint nicht eindeutig geklärt. Da im Bereich der Blattpolster solcher Stammstücke Parichnosmale nachgewiesen werden konnten (Daber \& Kahlert 1970), sind sie wohl tatsächlich der Gattung Lepidodendron oder sogar einer neuen Gattung zuzuordnen.

Die Frage, ob bei Asolanus eine Ligula vorhanden war, läßt sich auch am hier vorliegenden Material aufgrund der relativ ungünstigen Erhaltungsbedingungen nicht eindeutig entscheiden. Allerdings findet sich, wie auch bei vielen anderen Stücken, die bisher von Asolanus beschrieben wurden, am oberen Rand der Blattnarbe median eine Einbuchtung, oft mit v-förmigem Umriß, die höchstwahrscheinlich einer Ligulargrube entspricht (Taf. 1: 5).

Auf der vorliegenden fertilen Achse konnten insgesamt drei morphologisch gleichartige Megasporen-Anhäufungen jeweils auf der adaxialen Seite der Sporophylle nachgewiesen werden. Sie sitzen frei verteilt zwischen weit über 20 noch ansitzend erhaltenen Sporophyllen, auf denen keine Sporenmassen mehr liegen. Da nur ein einzelnes und relativ kurzes fertiles Stück von 
Asolanus aufgesammelt werden konnte, ist der vollständige Bau des fertilen Achsenabschnitts nicht zu klären. Man darf aber vermuten, daß es sich um einen weitgehend reifen "Zapfen" handelt, aus dem die anderen Sporangien bereits herausgefallen sind. Eindeutig belegt werden kann das aber nicht. So wäre es auch denkbar, daß nur vereinzelt Sporangien ausgebildet wurden und die restliche Achse steril blieb. Zwischen den breiten und stumpf gerundeten Blättern würden dann vereinzelt nicht weiter differenzierte Sporophylle mit ansitzenden Sporangien ausgebildet werden.

Das frei verteilte Auftreten fertiler Komplexe an sonst sterilen Achsen ist bei den Lepidophyten bekannt, wie z. B. bei Pinakodendron. Diese Gattung wird in die Nähe von Asolanus gestellt, ist von dieser aber deutlich $u$. a. durch den Bau der fertilen Organe getrennt, da bei letzterer nur eine einzelne Tetrade auf dem Sporophyll ausdifferenziert wird (Amerom \& Gaipl 1991).

Vergleichbar gebaut ist Sporangiostrobus, die fertile Achse von Bodeodendron (Wagner 1989), beides Synonyme von Omphalophloios White (Brousmiche-Delcambre et al. 1995). Auch bei dieser Lepidophyte sind keine Parichnosmale nachweisbar, das Vorhandensein einer Ligula ist nicht sicher (Wagner \& Spinner 1976). Die distalen Abschnitte der Pflanze werden insgesamt fertil, wobei die Sporangien mit den schmalen und langen Sporophyllen direkt am Stamm ansitzen. Diese Lepidophyte gehört damit offensichtlich zur selben, isoliert stehenden Gruppe wie Asolanus, ist von dieser Gattung aber eindeutig durch den Bau der Blattpolster und der Blätter/Sporophylle getrennt.

Lesquereux (1880: 445) beschreibt Sporophylle unter dem Namen Lepidostrobus foliaceus (im Atlas von 1879, Taf. 69: 8 als Lepidophyllum foliaceum bezeichnet), die denen von $A$. camptotaenia ähneln. Daneben bildet er die Zeichnung eines "Lepidophloios-Zapfen-Fragmentes" ab (Taf. 68: 6), bei dem auf schmalen, quergestreckten Marken breite, kurze Blätter mit kräftiger Mittelader ansitzen. An Stellen, wo die Blätter weggebrochen sind, kommen Sporenanhäufungen von abgerundet-rechteckigem Umriß zu Vorschein. Hier liegt ebenfalls die Vermutung nahe, daß es sich um $A$. camptotaenia handelt. Auch bei den als Lepidophyllum truncatum abgebildeten aber nicht näher beschriebenen „?Sporangien“ (Taf. 69: 9, 10) dürfte es sich eher um Sporophylle vom Asolanus-Typ handeln.

Einzelne blattartige Organe, die den Sporophyllen von $A$. camptotaenia entsprechen, wur- den häufiger gefunden und $\mathbf{u}$. a. von Bureau (1914: 165) zu Gymnostrobus salisburyi gestellt, was Jongmans (1930: 379) bereits als falsch erkannte. Trotzdem stellte Bell (1943: 95) vergleichbare Organe unter diesem Namen zu einer Stigmarie in „Gymnostrobus-Erhaltung“.

Pant (1956: 421) belegt noch einmal ausführlich die Auffassung, daß Gymnostrobus ein Erhaltungszustand von Stigmaria ist. Bell (1962: 57) akzeptierte diese Auffassung so nicht und benutzte die Gattung Gymnostrobus für seine neue Art G. wilsoni. Dabei vermutete er bereits eine Zugehörigkeit zur Gattung Asolanus. Da die Achse mit den seitlich ansitzenden Sporophyllen nur in einer Knorria-artigen Erhaltung vorlag, konnte er den endgültigen Beweis jedoch nicht antreten.

Im Gesamthabitus ähnlich gebaute Sporophylle beschreiben Stepanek \& Vogellehner (1985) aus dem Namur oder tiefen Westfal des Schwarzwaldes unter dem Namen Lepidostrobophyllum diersburgense. Diese Zapfenschuppen sind allerdings spatelförmig gebaut und bei einer Länge von $26 \mathrm{~mm}$ und einer Breite von durchschnittlich 4,5 mm deutlich länger und schmaler. Stepanek \& Vogellehner weisen darauf hin, daß kein Pedicel zu existieren scheint, nur bei wenigen Stücken sind Strukturen nachweisbar, die auf ein Pedicel hinweisen, das in die Spreite der Zapfenschuppe integriert ist, ähnlich wie bei den vorliegenden Sporophyllen von $A$. camptotaenia. Vergleichbar erscheint auch die ausgeprägt breite Mittelader im Verhältnis zur Gesamtbreite von L. diersburgense sowie das stumpf gerundete, distale Ende der Zapfenschuppen. Auf und direkt neben diesen Zapfenschuppen konnten trilete Sporen mit einem Durchmesser von etwa $0,5 \mathrm{~mm}$ nachgewiesen werden, was in etwa auch den Dimensionen der Megasporen vom Piesberg entspricht.

Stepanek \& Vogellehner (1985: 84) vergleichen ihr Material mit Lepidophyllum volkmannianum (Hartung 1938: 126) vor allem aufgrund des Basalteils der Sporophylle. Der Gesamthabitus von L. volkmannianum entspricht allerdings ansonsten überhaupt nicht dem Bau der Zapfenschuppen von $L$. diersburgense. So weisen die Sporophylle von $L$. volkmannianum eine deutliche Gliederung in einen breiten Basalteil und eine schmale, spitz auslaufende Spreite auf, wodurch sie im Gegensatz zu $L$. diersburgense und den Sporophyllen von $A$. camptotaenia den typisch lanzettförmigen Lepidophyllen-Habitus zeigen.

Mit L. diersburgense in organischem Zusammenhang stehende Achsenreste konnten bisher 
nicht nachgewiesen werden. Zusammen mit diesen Lepidostrobophyllen finden sich allerdings häufiger die Gattungen Sigillaria, Lepidodendron, Sublepidophloios und eine Art der Gattung Lepidophloios mit sehr weit auseinanderstehenden Blattpolstern. Hier könnte man vielleicht eine Form vermuten, die möglicherweise zur Gattung Asolanus überleitet.

Zweifelsfrei an Stamm- oder Achsenresten ansitzende sterile Blätter sind bisher nicht nachgewiesen worden. Aber bereits Weiss \& Sterzel (1893: 72; Taf. 4: 24) bilden ein Stück ab, bei dem mehrere schmale Blätter vom Lepidophyllum-Habitus direkt auf einzelne Blattpolster zulaufen. Ein unwiderlegbarer Beweis für den direkten Zusammenhang ist nicht zu führen, wie auch Daber \& Kahlert (1970: 349) schreiben, die den Abbildungsbeleg nachuntersuchten. Nach Remy \& Remy (1959) sollen 4 cm lange, schmale Blätter zu Asolanus gehören. Crookall (1964) beschreibt die Blätter ebenfalls als lang, "grass-like", an der Basis 5 oder $6 \mathrm{~mm}$ breit, nach vorne gleichmäßig zusammenlaufend, mit einer einzigen Mittelader. Solche Blätter finden sich auch bei dem Material vom Piesberg in unmittelbarer Nachbarschaft zu einem Stammstück von A. camptotaenia. Ein organischer Zusammenhang konnte auch hier nicht eindeutig bewiesen werden. Ein direktes Ansitzen darf aber doch angenommen werden, da mehrere Blätter direkt auf Blattpolster zulaufen und dann im Bereich der Blattpolster verschwinden. Zudem konnten bei mehreren Grabungskampagnen keine anderen Lepidophytenreste gefunden werden, zu denen diese typischen Lepidophyllen gehören könnten.

\section{Taxonomische Stellung}

Die von Daber \& Kahlert (1970: 351) vorgenommene Herabstufung der Gattung Asolanus zu einer Untergattung erscheint nicht unbedingt zwingend. Die Autoren sprechen von dem Auftreten atavistischer Merkmale bei Asolanus. Dadurch sei die Zugehörigkeit zur Gattung Lepidodendron belegt. Nun setzt diese Beurteilung aber voraus, daß es sich bei dem beschriebenen Stück eindeutig um Asolanus camptotaenia handelt. Das aus den Wettiner Schichten (Stefan) von Plötz stammende Stück weist nicht das typische Längen-Breiten-Verhältnis der Blattnarben von Asolanus auf. Auch ist die Skulpturierung der Felder zwischen den Blattpolstern auffallend grob und kräftig, fast wulstig. Besonders auffällig sind aber die seitlich auf dem Blattpolster stehenden Parichnosmale, die weder bei den vorliegenden Stücken noch auf älteren Abbildungen von Asolanus auch nur andeutungsweise nachweisbar sind, deren Fehlen auch in der Literatur angegeben wird (Renier et al. 1938; Crookall 1964).

Der Bau eines jetzt erstmalig nachgewiesenen fertilen Sproßteiles bestätigt daher eher die alte Annahme, daß es sich bei Asolanus um ein relativ isoliertes Taxon innerhalb der Lepidophyta handelt. Fehlende deutliche Differenzierungen an den Trag- oder Schutzorganen und der sehr lockere Aufbau des fertilen Achsenabschnitts sind Merkmale, die die Gattung Asolanus noch deutlicher als bisher von der Gruppe der „klassischen" Lepidophyten trennt.

\section{Stratigraphische Verbreitung}

Lejal-Nicol (1985) gibt $A$. camptotaenia aus dem tiefen Namur von Algerien an, doch handelt es sich hier um einen nicht näher bestimmbaren Pflanzenrest. Gesichert ist das stratigraphisch tiefste Auftreten von $A$. camptotaenia mit dem oberen Teil der Hendrik-Gruppe aus dem Limburger Karbon, der dem höheren Anteil des unteren Westfal B entspricht (Hirmer 1940: 167). Drägert (1964: 40; Taf. 11: 5) erwähnt $A$. camptotaenia aus dem tiefen Westfal B (mittlere Essener Schichten) des Ruhrgebietes, doch zeigt das sehr kleine abgebildete Stück keine eindeutigen Merkmale, die eine solche Bestimmung bestätigen würden. Im Westfal C/D wird die Art häufiger (Josten 1991: 152; Zeiller 1888: 588), um dann im nordeuropäischen Raum langsam auszuklingen. Nach Grand'Eury in Weiss \& Sterzel (1893: 75) ist A. camptotaenia im Stefan des Gard-Beckens (Süd-Frankreich) noch häufig.

Während Crookall (1964: 349) für das englische Karbon das seltene Auftreten von A. camptotaenia hervorhebt, spricht Hirmer (1940: 177, 227) für das Limburger Karbon von einer „besonders bezeichnenden Art und für das Piesberger Karbon sogar von ,einer Charakterpflanze 1. Ranges". Auch Gothan \& Weyland (1973: 169) sprechen von einem nicht seltenen Auftreten dieser Gattung im euramerischen Karbon und Daber \& Kahlert (1970: 360) bezeichnen die „Formgattung als relativ häufig“. So wurde $A$. camptotaenia im Piesberg unter dem Namen Lepidodendron barbatum bereits von Roemer (1864: 40) als häufig über Flöz Mittel angegeben. Neben Weiss \& Sterzel (1893) konnte Josten 
(1991: 152) diese Art dort vor allem im Hangenden von Flöz Johannissteine bis in die letzte Zeit nachweisen. In den entsprechenden Sammlungen finden sich daher konzentriert viele Stammstücke von Asolanus als etwas "Besonderem“, was "mitnehmenswert" ist. Bei den eigenen Aufsammlungen am Piesberg sind trotz jahrelanger, intensiver Sammeltätigkeit nur wenige Reste dieser Gattung aufgefunden worden.

Die hier beschriebenen Stücke von A. camptotaenia stammen aus dem Hangenden des höchsten am Piesberg aufgeschlossenen Flözes, dem Flöz Itterbeck, d. h. nach dem bisherigen Kenntnisstand (Florenzusammensetzung, Datierung nach dem „Itterbeckhorizont") aus dem tieferen Westfal D (Abb. 1).

\section{Danksagung}

Besonders verdienstvoll war die Hilfe von Herrn M. Born, München, bei der Profilaufnahme der Abfolgen um Flöz Itterbeck in der Mülldeponie Osnabrück. Herr Prof. W. Remy $(\dagger)$, Münster begleitete noch die Anfänge der Arbeit mit hilfreichen Hinweisen und Diskussionsbeiträgen. Frau W. Harre und Frau E. Siebert, Berlin, ist für ihre bewährte Unterstützung bei den Photoarbeiten und der Erstellung der Rekonstruktionszeichnungen zu danken.

\section{Literatur}

Amerom, H. W. J. van \& Gaipl, R. 1991. Ein Beitrag zur Ikonographie von Pinakodendron musivum Weiss 1893. - Neues Jahrbuch für Geologie und Paläontologie, Abhandlungen $183(1-3)$ : 217-235.

Bell, W.A. 1943. Carboniferous Rocks and Fossil Floras of Northern Nova Scotia. - Memoirs of the Geological Survey of Canada 238: $276 \mathrm{pp}$.

- 1962. Flora of Pennsylvanian Pictou Group of New Brunswick. - Bulletin of the Geological Survey of Canada 87: $71 \mathrm{pp}$.

Brousmiche-Delcambre, C; Coquel, R. \& Wagner, R.H. 1995. Nouvelle interprétation du genre Omphalophloios White, 1898 (Lycophyte primitive). - Comptes rendues de l'Académie des Sciences Paris 321, sér. II a: 179-184.

Bureau, M.E. 1914. Bassin de la Basse Loire. Flores Fossiles. - Études des Gîtes Minéraux de la France, 1914: 403 pp.

Chaloner, W.G. 1967. Lycophyta. In Boureau, E. (ed.): Traité de Paléobotanique. II. Bryophyta, Psilophyta, Lycophyta: 437-802. - Paris (Masson et Cie.).

Crookall, R. 1964. Fossil Plants of the Carboniferous Rocks of Great Britain. - Memoirs of the Geological Survey of Great Britain, Palaeontology 4 (3): 217-354.

Daber, R. \& Kahlert, E. 1970. Lepidodendron (Asolanus) camptotaenia (Wood, 1860) comb. n. - Paläontologische Abhandlungen, B, Paläobotanik 3 (3-4): 349--354.

Drägert, K. 1964. Pflanzensoziologische Untersuchungen in den Mittleren Essener Schichten des nördlichen Ruhrgebietes. - Forschungsberichte des Landes Nordrhein-Westfalen 1363: $270 \mathrm{pp}$.

Gothan, W. \& Weyland, H. 1973. Lehrbuch der Paläobotanik: 594 pp. - Berlin (Akademie-Verlag).

Gothan, W. \& Remy, W. 1957. Steinkohlenpflanze: 248 pp. Essen (Glückauf).
Hartung, W. 1938. Flora und Altersstellung des Karbons von Hainichen-Ebersdorf und Borna bei Chemnitz (Erzgeb. Becken). - Abhandlungen des Sächsischen Geologischen Landesamtes 18: $140 \mathrm{pp}$.

Hirmer, M. 1940. Die Pflanzen des Karbon und Perm und ihre stratigraphische Bedeutung. Teil II: Die OberkarbonFloren der paralischen Becken des westlichen MittelEuropa. - Palaeontographica B 84: 153-269.

Hörich, O. 1920. Über Protasolanus, eine neue Lepidophytengattung aus dem deutschen Culm und über die Gattung Asolanus Wood. - Jahrbuch der preussisch geologischen Landesanstalt für 1919: 434 - 459.

Jongmans, W. 1930. Fossilium Catalogus. II: Plantae, 16: Lycopodiales III: 329-650 - Berlin (W. Junk).

Josten, K.-H. 1991. Die Steinkohlen-Floren Nordwestdeutschlands. - Fortschritte in der Geologie von Rheinland und Westfalen 36: $434 \mathrm{pp}$.

Josten, K.-H.; Köwing, K. \& Rabitz, A. 1984. Oberkarbon. In Klassen, H. (ed.): Geologie des Osnabrücker Berglandes: 7-78, 20 Abb., 4 Taf., 1 Tab. - Osnabrück (Naturwissenschaftliches Museum).

Kidston, R. 1911. Les végétaux houillers recueillis dans le Hainaut Belge. - Mémoires du Musée Royal d'Histoire Naturelle de Belgique 4: 282.

Knauff, W.; Köwing, K. \& Rabitz, A. 1971. Der erste Nachweis von Horizonten mit Foraminiferen im Westfal D von Nordwestdeutschland. - Fortschritte in der Geologie von Rheinland und Westfalen 18: 257-262.

Koehne, W. 1904. Sigillariae medulla (Marksteinkern von Sigillaria). In Potonié, H. (ed.): Abbildung und Beschreibung fossiler Pflanzen, 2 (37): 1-13.

Lejal-Nicol, A. 1985. Megafloras. In Wagner, R.H.; Winkler Prins, C. F. \& Granados, L.F. (ed.): The Carboniferous of the World. II. IUGS Publication 20: 386-391. - Madrid (Instituto Geologico y Minero de Espana).

Lesquereux, L. 1879. Attas of the Coal Flora of Pennsylvania and of the Carboniferous Formation throughout the United States. - 2. Geological Survey of Pennsylvania, Report of Progress, P.

- 1880. Description to the Coal Flora of the Carboniferous Formation in Pennsylvania and troughout the United States, 2 (1). - 2. Geological Survey of Pennsylvania, Report of Progress, P: $694 \mathrm{pp}$.

Pant, D. D. 1956. On two compressed Palaeozoic axes: Stigmaria ficoides in the Gymnostrobus condition and Vertebraria indica. - Annals of Botany, n.s. 20: 419-429.

Remy, W. \& Remy, R. 1959. Pflanzenfossilien. Ein Führer durch die Flora des limnisch entwickelten Paläozoikums: 285 pp. - Berlin (Akademie-Verlag).

Renier, A.; Stockmans, F.; Demanet, F. \& Van Straelen, V. 1938. Flore et Faune Houillères de la Belgique: 317 pp. Bruxelles (Musée Royale d'Histoire Naturelle de Belgique).

Roehl, E. von 1868. Fossile Flora der Steinkohlen-Formation Westphalens, einschliesslich Piesberg bei Osnabrück. Palaeontographica 18 (1-6): 1-192.

Roemer, F. A. 1864. Beiträge zur geologischen Kenntnis des nordwestlichen Harzgebirges. - Palaeontographica 9: $1-46$.

Stepanek, I. \& Vogellehner, D. 1985. Die Flora des unteren Oberkarbons von Diersburg-Berghaupten bei Offenburg (Mittlerer Schwarzwald) II: Lycopodiatae (Sigillariaceae und Lepidodendraceae). - Berichte der Naturforschenden Gesellschaft zu Freiburg 75: 71-90.

Vetter, P. 1968. Géologie et Paléontologie des Bassins houillers de Decazevile, de Figeac et du Détroit de Rodez. II. Étude paléontologique, 194 pp. - Aurillac (Imprimerie Moderne).

Vogellehner, D. 1967. Die Flora des Stefans an der Hohengeroldseck bei Lahr (Mittlerer Schwarzwald) II. Lycophyta (Lepidophytales). - Berichte der Naturforschenden Gesellschaft zu Freiburg 57: 245-266.

Wagner, R. H. 1989. A late Stephanian forest swamp with Sporangiostrobus fossilized by volcanic ash fall in the $\mathbf{P u}-$ 
ertollano Basin, Central Spain. - International Journal of Coal Geology 12: 523-552.

Wagner, R. H. \& Spinner, E. 1976. Bodeodendron, tronc associé B Sporangiostrobus. - Comptes Rendues de l'Académie des Sciences de Paris D 282: 353-356.

Weiss, Ch. E. \& Sterzel, T. 1893. Beiträge zur fossilen Flora, V. Die Sigillarien der preussischen Steinkohlen- und Rothliegenden-Gebiete. II. Die Gruppe der Subsigillarien. - Abhandlungen der preussisch geologischen Landesanstalt, N.F. 2: 255 pp.

Wood, H. C. 1869 . A Contribution to the Knowledge of the Flora of the Coal Period in the United States. - Trans- actions of the American Philosophical Society, N.S. 13: $341-349$.

Zeiller, R. 1886. Bassin houiller de Valenciennes. Description de la flore fossile. Atlas. - Études des Gîtes Mineraux de France 1886: 94 pls.

- 1888. Bassin houiller de Valenciennes. Description de la flore fossile. - Études des Gîtes Mineraux de France 1888: $731 \mathrm{pp}$

- 1906. Bassin houiller et Permien de Blanzy et du Creusot, II. Flore Fossile, Atlas. - Études des Gîtes Mineraux de France 1906: $51 \mathrm{pls}$. 